\title{
THE MULTIPLICATION OPERATOR, ZERO LOCATION AND ASYMPTOTIC FOR NON-DIAGONAL SOBOLEV NORMS
}

\author{
ANA PORTILLA ${ }^{\dagger}$, JOSÉ M. RODRÍGUEZ $^{\dagger}$, AND EVA TOURÍS ${ }^{\dagger}$
}

\begin{abstract}
In this paper we are going to study the zero location and asymptotic behavior of extremal polynomials with respect to a generalized non-diagonal Sobolev norm in which the product of the function and its derivative appears. The orthogonal polynomials with respect to this Sobolev norm are a particular case of those extremal polynomials. The multiplication operator by the independent variable is the main tool in order to obtain our results.

Key words and phrases: Multiplication operator; zero location; asymptotic; weight; Sobolev orthogonal polynomials; extremal polynomials; weighted Sobolev spaces.
\end{abstract}

\section{INTRODUCTION.}

Weighted Sobolev spaces are an interesting topic in many fields of Mathematics. In the classical book [16] we can find the point of view of Partial Differential Equations. We are mainly interested in the relationship between this topic and Approximation Theory in general, and Sobolev orthogonal polynomials in particular.

Sobolev orthogonal polynomials have been more and more investigated in recent years. In particular, in [13] and [14], the authors showed that the expansions with Sobolev orthogonal polynomials can avoid the Gibbs phenomenon which appears with classical orthogonal series in $L^{2}$.

In [27], [28], [29] and [30] the authors developed a theory of general Sobolev spaces with respect to measures in the real line, in order to apply it to the study of Sobolev orthogonal polynomials.

Sobolev orthogonal polynomials on the unit circle and, more generally, on curves is a topic of recent and increasing interest in approximation theory; see, for instance, [4] and [11] (for the unit circle) and [23] and [2] (for the case of Jordan curves). The papers [1], [2], [4], [11], [20] and [22] deal with Sobolev spaces on curves and more general subsets of the complex plane.

One of the central problems in the theory of Sobolev orthogonal polynomials is to determine its asymptotic behavior. In [21] the authors show how to obtain the $n$-th root asymptotic of Sobolev orthogonal polynomials if the zeros of these polynomials are contained in a compact set of the complex plane. Although the uniform bound of the zeros of orthogonal polynomials holds for every measure with compact support in the case without derivatives, it is an open problem to bound the zeros of Sobolev orthogonal polynomials. The boundedness of the zeros is a consequence of the boundedness of the multiplication operator $M f(z)=z f(z)$; in fact, the zeros of the Sobolev orthogonal polynomials are contained in the disk $\{z:|z| \leq 2\|M\|\}$ (see Theorem 2.1).

In [1], [28], [30], [31] and [32], there are some answers to the question stated in [21] about some conditions for $M$ to be bounded: the more general result on this topic is [1, Theorem 8.1] which characterizes in a simple way (in terms of equivalent norms in Sobolev spaces) the boundedness of $M$ for the classical "diagonal" case

$$
\|q\|_{W^{k, p}\left(\mu_{0}, \mu_{1}, \ldots, \mu_{N}\right)}:=\left(\sum_{k=0}^{N}\left\|q^{(k)}\right\|_{L^{p}\left(\mu_{k}\right)}^{p}\right)^{1 / p}
$$

(see Theorem 2.2 below, which is [1, Theorem 8.1] in the case $N=1$ ). The rest of the papers mention several conditions which guarantee the equivalence of norms in Sobolev spaces, and consequently, the boundedness of $M$.

Date: June 26, 2009

† Supported in part by three grants from M.E.C. (MTM 2006-13000-C03-02, MTM 2006-11976 and MTM 2007-30904-E) and by a grant from U.C.III M./C.A.M. (CCG07-UC3M/ESP-3339), Spain. 
In [20], the authors study the asymptotic behavior of extremal polynomials with respect to the following "non-diagonal" Sobolev norms. Given a finite Borel measure $\mu$ with compact support $S(\mu)$ consisting of infinitely many points in the complex plane, let us consider the diagonal matrix $\Lambda:=\operatorname{diag}\left(\lambda_{j}\right), 0 \leq j \leq N$, with $\lambda_{j}$ positive $\mu$-almost everywhere measurable functions, and $U:=\left(u_{j k}\right), 0 \leq j, k \leq N$, a matrix of measurable functions such that the matrix $U(x)=\left(u_{j k}(x)\right), 0 \leq j, k \leq N$, is unitary $\mu$-almost everywhere. If $V:=U \Lambda U^{*}$, where $U^{*}$ denotes the transpose conjugate of $U$ (note that then $V$ is a positive definite matrix), and $1 \leq p<\infty$, we define the Sobolev norm

$$
\begin{aligned}
\|q\|_{W^{N, p}(V \mu)} & :=\left(\int\left[\left(q, q^{\prime}, \ldots, q^{(N)}\right) V^{2 / p}\left(q, q^{\prime}, \ldots, q^{(N)}\right)^{*}\right]^{p / 2} d \mu\right)^{1 / p} \\
& =\left(\int\left[\left(q, q^{\prime}, \ldots, q^{(N)}\right) U \Lambda^{2 / p} U^{*}\left(q, q^{\prime}, \ldots, q^{(N)}\right)^{*}\right]^{p / 2} d \mu\right)^{1 / p} .
\end{aligned}
$$

It is not difficult to verify that under the assumptions imposed, $\|\cdot\|_{W^{N, p}(V \mu)}$ defines a norm on the space of polynomials $\mathbb{P}$. If $U$ is not the identity matrix $\mu$-almost everywhere, then (1) defines a generalized nondiagonal Sobolev norm in which the product of derivatives of different order appears.

We say that $q_{n}=z^{n}+a_{n-1} z^{n-1}+\cdots+a_{1} z+a_{0}$ is an $n$-th monic extremal polynomial with respect to the norm (1) if

$$
\left\|q_{n}\right\|_{W^{N, p}(V \mu)}=\inf \left\{\|q\|_{W^{N, p}(V \mu)}: q=z^{n}+b_{n-1} z^{n-1}+\cdots+b_{1} z+b_{0}, b_{j} \in \mathbb{C}\right\} .
$$

It is clear that there exists at least an $n$-th monic extremal polynomial. Furthermore, it is unique if $1<p<\infty$ (see e.g. [5, pp. 22-23]). If $p=2$, then the $n$-th monic extremal polynomial is precisely the $n$-th monic Sobolev orthogonal polynomial with respect to the inner product corresponding to (1).

The following is one of the basic results of [20].

Theorem 1.1. ([20, Theorem 1]) Let $S(\mu)$ be compact and $1 \leq p<\infty$. Assume that there exists a constant $C$ such that

$$
\lambda_{j} \leq C \lambda_{k}, \quad 0 \leq j, k \leq N
$$

$\mu$-almost everywhere. Let $\left\{q_{n}\right\}_{n \geq 0}$ be a sequence of extremal polynomials with respect to (1). Then the zeros of the polynomials in $\left\{q_{n}\right\}_{n \geq 0}$ are uniformly bounded in the complex plane.

This result is interesting since it allows the authors to obtain the asymptotic behavior of extremal polynomials (see [20, Theorems 2 and 6]). To require compact support for $\mu$ is a natural hypothesis: if $S(\mu)$ is not bounded, then we can not expect to have zeros uniformly bounded, even in the classical case (orthogonal polynomials in $L^{2}$ ).

If we are interested in such a general result as Theorem 1.1, then the definition of the weight matrix $V$ as $V:=U \Lambda U^{*}$ considered in Theorem 1.1 is the most appropriate. The main aim of our paper is to obtain the same thesis as in Theorem 1.1, but with hypotheses directly on the matrix $V$ (rather than on the diagonal matrix $\Lambda$ which appears in its factorization); in exchange for a certain loss of generality, we require weaker hypothesis than (2).

Since a wide majority of works about Sobolev spaces (both pure and applied) are focused on the case $N=1$, we will assume that this is the situation throughout the current paper. That is why we consider directly the weight matrix $V$ as

$$
V:=\left(\begin{array}{cc}
a & b \\
\bar{b} & c
\end{array}\right)
$$

where $a, b$ and $c$ are measurable functions, and $V$ is a positive definite matrix $\mu$-almost everywhere. We say that it is elliptic if $|b|^{2} \leq(1-\varepsilon) a c, \mu$-almost everywhere for some fixed $0<\varepsilon \leq 1$. Notice that this is not a very restrictive condition, since $V$ is a positive definite matrix and therefore $|b|^{2}<a c$. In fact, ellipticity is a weaker condition than (2) (see Proposition 2.10). Furthermore, note that this ellipticity condition is much simpler and weaker than the commonly used in PDE's: the function $b$ takes real values and there exist constants $A$ and $B$ verifying

$$
A\left(\xi_{1}^{2}+\xi_{2}^{2}\right) \leq a(x) \xi_{1}^{2}+2 b(x) \xi_{1} \xi_{2}+c(x) \xi_{2}^{2} \leq B\left(\xi_{1}^{2}+\xi_{2}^{2}\right),
$$

for every $\left(\xi_{1}, \xi_{2}\right) \in \mathbb{R}^{2}$ and $x \in \mathbb{R}$; in particular, this latter condition implies $A \leq a(x), c(x) \leq B$ for every $x \in \mathbb{R}$. 
A particular case (simpler to state) of our main result (Theorem 2.7) is the following.

Theorem 1.2. Let $S(\mu)$ be compact, $V$ elliptic, and $2 \leq p<\infty$. Assume that there exists a constant $C$ such that

$$
c \leq C a,
$$

$\mu$-almost everywhere. Let $\left\{q_{n}\right\}_{n \geq 0}$ be the sequence of extremal polynomials with respect to (1). Then the zeros of the polynomials in $\left\{q_{n}\right\}_{n \geq 0}$ are uniformly bounded in the complex plane.

In our context there is no such thing as the usual three term recurrence relation for orthogonal polynomials in $L^{2}$. Therefore it is very complicated to find an explicit expression for the extremal polynomial of degree $n$. Hence, it is especially important to count with an asymptotic estimate for the behavior of extremal polynomials.

As an application of Theorem 2.7, we can deduce the asymptotic behavior of extremal polynomials (see Theorems 3.1 and 3.2). In particular, we obtain the $n$-th root and the zero counting measure asymptotics both of those polynomials and their derivatives of any order. The asymptotics of the $n$-th root is a classical problem in the theory of orthogonal polynomials (see e.g. [20], [21], [22], [34], [35]).

Furthermore, in Theorem 3.2 we find the following asymptotic relation:

$$
\lim _{n \rightarrow \infty} \frac{q_{n}^{(j+1)}(z)}{n q_{n}^{(j)}(z)}=\int \frac{d \omega_{S(\mu)}(x)}{z-x}
$$

for any $j \geq 0$.

We also have similar results for the case $1 \leq p<2$; however, since they are not so simple to state as the results for $2 \leq p<\infty$ (see Theorem 2.4 and the remark after its proof), we prefer not to announce them in this section.

The advantages of our results with respect to earlier results (see Theorem 1.1 and Theorem 2.2) are mainly the following:

- We generalize the characterization of the boundedness of the multiplication operator in the diagonal case appearing in Theorem 2.2 to the non-diagonal case.

- The condition (3) in Theorem 1.2 refers to the coefficients of $V$ instead of to its eigenvalues, which is much more natural: although we also have the need to factor out the matrix $V$, we do it just inside our proofs, but not in the statements of the theorems.

- We have replaced the double inequality $\lambda_{0} \leq C \lambda_{1}, \lambda_{1} \leq C \lambda_{0}$ by the single one $c \leq C a$ (see Theorem 1.2), which is a much weaker condition, and by the ellipticity condition. On the one hand, this ellipticity condition is just a bit more restrictive that the hypothesis of $V$ being a positive definite matrix; on the other hand, in Proposition 2.10 we show that (2) implies the ellipticity of $V$, and that the converse is false.

- We have even a stronger version of Theorem 1.2 (see Theorem 2.8).

- Note that the boundedness of the zeros of extremal polynomials is a direct consequence of the boundedness of the multiplication operator $M$. Our Theorem 2.7 is sharp in the sense that it gives a characterization of the boundedness of $M$.

- Besides, Theorem 2.7 is also sharp for the "diagonal case" $b=0$, whereas the condition (2) in Theorem 1.1, although useful, is not sharp at all for the diagonal case. Note that condition (2) is the best possible in the context of [20], since starting from the factorization of $V$ it is not possible to distinguish between the roles of the eigenvalues (the choice of the names $\lambda_{0}$ and $\lambda_{1}$ is arbitrary).

As an application that shows the power of our results, we include a very natural and general example. It makes clear that our theorems can be applied for a much wider range of cases than Theorems 1.1 and 2.2 (see the example at the end of Section 2).

As we have mentioned before, the main idea of this paper is to change the point of view of Theorem 1.1. We will focus directly on the matrix $V$ instead of on its factorization. This will allow us to obtain a sharp result on equivalent norms in Sobolev spaces, and as a consequence of it, we will get the boundedness of zeros and the asymptotic behavior of extremal polynomials. 
The outline of the paper is as follows. Section 2 is devoted to the results on equivalent norms, and their applications to the study of the multiplication operator and the location of zeros of extremal polynomials. In Section 3 we obtain the results on asymptotic of extremal polynomials.

Acknowledgements. We would like to thank the referees for their careful reading of the manuscript and for some helpful suggestions, which contributed to improve the paper.

\section{The MUltiplicAtion OPERATOR.}

As we have mentioned in the introduction, given a finite Borel measure $\mu$ with compact $\operatorname{support} S(\mu)$ consisting of infinitely many points in the complex plane, and a positive definite matrix $\mu$-almost everywhere

$$
V:=\left(\begin{array}{cc}
a & b \\
\bar{b} & c
\end{array}\right)
$$

for any $1 \leq p<\infty$ and any polynomial $q$, we define the Sobolev norm of $q$ in $W^{1, p}(a \mu, c \mu)$ by

$$
\|q\|_{W^{1, p}(a \mu, c \mu)}:=\left(\int\left(a|q|^{p}+c\left|q^{\prime}\right|^{p}\right) d \mu\right)^{1 / p} .
$$

It is obviously much easier to deal with this norm than with the one defined in (1). Therefore, one of our main goals is to provide weak hypotheses to guarantee the equivalence of both norms (see Theorem 2.3).

In order to bound the zeros of polynomials, one of the most successful strategies has certainly been to bound the multiplication operator by the independent variable $M f(z)=z f(z)$, where

$$
\|M\|:=\sup \left\{\|z q(z)\|_{W^{1, p}(V \mu)}:\|q\|_{W^{1, p}(V \mu)}=1\right\} .
$$

Regarding this issue, the following result is known.

Theorem 2.1. ([20, Theorem 3]) Let $S(\mu)$ be compact and $1 \leq p<\infty$. Let $\left\{q_{n}\right\}_{n \geq 0}$ be a sequence of extremal polynomials with respect to (1). Then the zeros of $\left\{q_{n}\right\}_{n \geq 0}$ lie in the bounded disk $\{z:|z| \leq 2\|M\|\}$.

It is also known a simple characterization of the boundedness of $M$.

Theorem 2.2. ([1, Theorem 8.1]) The multiplication operator is bounded in $W^{1, p}(a \mu, c \mu)$ if and only if the following condition takes place:

$$
\text { The norms in } W^{1, p}((a+c) \mu, c \mu) \text { and } W^{1, p}(a \mu, c \mu) \text { are equivalent on } \mathbb{P} \text {. }
$$

It is clear that if there exists a constant $C$ such that $c \leq C a \mu$-almost everywhere, then (5) holds. In [31] and [32] some other very simple conditions implying (5) are shown.

We say that two functions $u, v$ are comparable (and we write $u \asymp v$ ) if there are positive constants $c_{1}, c_{2}$ such that $c_{1} v \leq u \leq c_{2} v$.

We prove now the announced result about the equivalence of the norms.

Theorem 2.3. If $V$ is elliptic, then $V^{2 / p}$ is also elliptic and the norms $W^{1, p}(V \mu)$ and $W^{1, p}(a \mu, c \mu)$ are equivalent on the space of polynomials for every $2 \leq p<\infty$.

Proof. Without loss of generality we can assume that $\varepsilon \in(0,1)$, since if $\varepsilon=1$ then $V$ is a diagonal matrix and the result is straightforward. The characteristic polynomial of $V$ is $\lambda^{2}-(a+c) \lambda+a c-|b|^{2}$, and it means that its two eigenvalues, respectively $\lambda_{+}=\lambda_{+}(z)$ and $\lambda_{-}=\lambda_{-}(z)$, are given by the formula:

$$
\lambda_{ \pm}=\frac{a+c \pm \sqrt{(a+c)^{2}-4\left(a c-|b|^{2}\right)}}{2}=\frac{a+c \pm \sqrt{(a-c)^{2}+4|b|^{2}}}{2} .
$$

On the one hand, it is obvious that:

$$
\lambda_{+}=\frac{a+c+\sqrt{(a-c)^{2}+4|b|^{2}}}{2} \geq \frac{a+c+|a-c|}{2}=\max \{a, c\} .
$$

On the other hand, taking into consideration that the matrix $V$ is positive definite, we know:

$$
\lambda_{+}=\frac{a+c+\sqrt{(a+c)^{2}-4\left(a c-|b|^{2}\right)}}{2} \leq a+c \leq 2 \max \{a, c\} .
$$


By (6) and (7) we conclude that $\lambda_{+} \asymp \max \{a, c\}$.

Next, we will do something similar for $\lambda_{-}$, the second eigenvalue. Notice that

$$
\lambda_{-}=\frac{a+c-\sqrt{(a-c)^{2}+4|b|^{2}}}{2} \leq \frac{a+c-|a-c|}{2}=\min \{a, c\} .
$$

In order to obtain a lower bound for $\lambda_{-}$let us define the following function:

$$
f(t):=1+t-\sqrt{1+t^{2}+(2-4 \varepsilon) t} .
$$

Since $\varepsilon \in(0,1), f^{\prime}(t) \neq 0$ for every $t \in[1, \infty)$. Furthermore, $f^{\prime}(1)=1-\sqrt{1-\varepsilon}>0$; hence, $f$ is increasing on the interval $[1, \infty)$.

On the other hand, using the fact that $V$ is elliptic,

$$
\frac{2 \lambda_{-}}{\min \{a, c\}}=\frac{a+c-\sqrt{(a-c)^{2}+4|b|^{2}}}{\min \{a, c\}} \geq \frac{a+c-\sqrt{a^{2}+c^{2}+(2-4 \varepsilon) a c}}{\min \{a, c\}} .
$$

If we define $t:=\max \{a, c\} / \min \{a, c\}$, the last term in the expression above can be written as $f(t)$. Using that $f$ is increasing on $[1, \infty)$, we deduce,

$$
\frac{2 \lambda_{-}}{\min \{a, c\}} \geq f(t) \geq f(1)=2(1-\sqrt{1-\varepsilon}) .
$$

And then,

$$
\lambda_{-} \geq(1-\sqrt{1-\varepsilon}) \min \{a, c\} .
$$

By (8) and (9) we conclude that $\lambda_{-} \asymp \min \{a, c\}$.

Let us define now

and the diagonal matrix

$$
\lambda_{0}:=\left\{\begin{array}{ll}
\lambda_{+} & \text {if } a \geq c, \\
\lambda_{-} & \text {if } a<c,
\end{array} \quad \lambda_{1}:= \begin{cases}\lambda_{-} & \text {if } a \geq c \\
\lambda_{+} & \text {if } a<c\end{cases}\right.
$$

$$
\Lambda:=\left(\begin{array}{cc}
\lambda_{0} & 0 \\
0 & \lambda_{1}
\end{array}\right) .
$$

Let $v_{0}$ be an eigenvector of $V$ corresponding to $\lambda_{0}$. Multiplying $v_{0}$ by an appropriate constant, we obtain an eigenvector $u_{0}$ with $\left\|u_{0}\right\|=1$ and such that its first coordinate is a real number. Notice that we can write:

$$
u_{0}=\left(\begin{array}{c}
\cos \theta \\
e^{i \varphi} \sin \theta
\end{array}\right)
$$

for some $\theta=\theta(z)$ and $\varphi=\varphi(z)$. We define now

$$
u_{1}=\left(\begin{array}{c}
-e^{-i \varphi} \sin \theta \\
\cos \theta
\end{array}\right)
$$

Note that $\left\|u_{1}\right\|=1$ and $\left\langle u_{0}, u_{1}\right\rangle=0$. Therefore, $u_{1}$ is an eigenvector of $V$ corresponding to $\lambda_{1}$, and the unitary matrix

$$
U=\left(\begin{array}{cc}
\cos \theta & -e^{-i \varphi} \sin \theta \\
e^{i \varphi} \sin \theta & \cos \theta
\end{array}\right)
$$

satisfies $V=U \Lambda U^{*}$. Consequently,

$$
V^{2 / p}=U \Lambda^{2 / p} U^{*}=:\left(\begin{array}{cc}
a_{p} & b_{p} \\
\bar{b}_{p} & c_{p}
\end{array}\right) .
$$

Now we want to show that $V^{2 / p}$ is also elliptic, i.e., that the inequality $\left|b_{p}\right|^{2} \leq(1-\varepsilon) a_{p} c_{p}$ holds $\mu$-almost everywhere.

In virtue of $V^{2 / p}=U \Lambda^{2 / p} U^{*}$ we have

$$
\left(\begin{array}{ll}
a_{p} & b_{p} \\
\bar{b}_{p} & c_{p}
\end{array}\right)=\left(\begin{array}{cc}
\cos \theta & -e^{-i \varphi} \sin \theta \\
e^{i \varphi} \sin \theta & \cos \theta
\end{array}\right)\left(\begin{array}{cc}
\lambda_{0}^{2 / p} & 0 \\
0 & \lambda_{1}^{2 / p}
\end{array}\right)\left(\begin{array}{cc}
\cos \theta & e^{-i \varphi} \sin \theta \\
-e^{i \varphi} \sin \theta & \cos \theta
\end{array}\right) .
$$


Performing the calculations, it holds

$$
\begin{aligned}
a_{p} & =\lambda_{0}^{2 / p} \cos ^{2} \theta+\lambda_{1}^{2 / p} \sin ^{2} \theta, \\
c_{p} & =\lambda_{0}^{2 / p} \sin ^{2} \theta+\lambda_{1}^{2 / p} \cos ^{2} \theta, \\
b_{p} & =\left(\lambda_{0}^{2 / p}-\lambda_{1}^{2 / p}\right) e^{-i \varphi} \cos \theta \sin \theta,
\end{aligned}
$$

and one can check that the inequality $\left|b_{p}\right|^{2} \leq(1-\varepsilon) a_{p} c_{p}$ is equivalent to

$$
\left(\frac{\lambda_{0}}{\lambda_{1}}\right)^{2 / p}+\left(\frac{\lambda_{1}}{\lambda_{0}}\right)^{2 / p} \leq 2 \frac{\sin ^{2} 2 \theta+(1-\varepsilon)\left(1+\cos ^{2} 2 \theta\right)}{\varepsilon \sin ^{2} 2 \theta},
$$

since $\left|b_{p}\right|^{2} \leq(1-\varepsilon) a_{p} c_{p}$ is equivalent to

$$
2 \lambda_{0}^{2 / p} \lambda_{1}^{2 / p} \sin ^{2} \theta \cos ^{2} \theta+(1-\varepsilon) \lambda_{0}^{2 / p} \lambda_{1}^{2 / p}\left(\sin ^{4} \theta+\cos ^{4} \theta\right) \geq \varepsilon\left(\lambda_{0}^{4 / p}+\lambda_{1}^{4 / p}\right) \sin ^{2} \theta \cos ^{2} \theta .
$$

Applying now the following trigonometric equalities

we have that

$$
\begin{aligned}
\sin ^{4} \theta+\cos ^{4} \theta & =\frac{1}{2}\left(1+\cos ^{2} 2 \theta\right), \\
\sin ^{2} \theta \cos ^{2} \theta & =\frac{1}{4} \sin ^{2} 2 \theta,
\end{aligned}
$$

$$
\frac{1}{2} \lambda_{0}^{2 / p} \lambda_{1}^{2 / p}\left(\sin ^{2} 2 \theta+(1-\varepsilon)\left(1+\cos ^{2} 2 \theta\right)\right) \geq \frac{\varepsilon}{4}\left(\lambda_{0}^{4 / p}+\lambda_{1}^{4 / p}\right) \sin ^{2} 2 \theta,
$$

and dividing both sides by $\lambda_{0}^{2 / p} \lambda_{1}^{2 / p}$ we get (11).

Since $V$ is elliptic, the inequality (11) is true for $p=2$; therefore it will be sufficient to check

$$
\left(\frac{\lambda_{0}}{\lambda_{1}}\right)^{2 / p}+\left(\frac{\lambda_{1}}{\lambda_{0}}\right)^{2 / p} \leq \frac{\lambda_{0}}{\lambda_{1}}+\frac{\lambda_{1}}{\lambda_{0}}
$$

for every $p \geq 2$. But this is true since

$$
F(t)=t+\frac{1}{t}-t^{2 / p}-\frac{1}{t^{2 / p}}=\frac{\left(t^{1+2 / p}-1\right)\left(t^{1-2 / p}-1\right)}{t} \geq 0
$$

for every $t>0$ and $p \geq 2$. Then, $V^{2 / p}$ is, indeed, elliptic.

We will show now that $a_{p} \asymp a^{2 / p}$ and $c_{p} \asymp c^{2 / p}$.

In what follows we assume that $a \geq c$; the proof in the other case is similar. Note that in this situation, $a \geq c$, we have already proved that $\lambda_{0}=\lambda_{+} \asymp a$ and $\lambda_{1}=\lambda_{-} \asymp c$.

We distinguish two cases.

(A) If $a / 2 \leq c \leq a$, then by (6), (7), (8) and (9) we deduce both that $a \leq \lambda_{+} \leq 2 a$ and $(1-\sqrt{1-\varepsilon}) c \leq$ $\lambda_{-} \leq c$. Since $a / 2 \leq c$, we conclude

$$
(1-\sqrt{1-\varepsilon}) \frac{a}{2} \leq \lambda_{-}, \lambda_{+} \leq 2 a .
$$

By (10), we have $\lambda_{-}^{2 / p} \leq a_{p}, c_{p} \leq \lambda_{+}^{2 / p}$. Then,

$$
\left(\frac{1-\sqrt{1-\varepsilon}}{2}\right)^{2 / p} a^{2 / p} \leq a_{p}, c_{p} \leq 2^{2 / p} a^{2 / p}
$$

and, in particular,

$$
\left(\frac{1-\sqrt{1-\varepsilon}}{2}\right)^{2 / p} c^{2 / p} \leq c_{p} \leq 4^{2 / p} c^{2 / p}
$$


(B) If $c<a / 2$ notice first that

$$
\begin{aligned}
& a=\lambda_{+} \cos ^{2} \theta+\lambda_{-}\left(1-\cos ^{2} \theta\right), \\
& c=\lambda_{+}\left(1-\cos ^{2} \theta\right)+\lambda_{-} \cos ^{2} \theta .
\end{aligned}
$$

Using these equalities, the fact that $V$ is positive definite and that $c<a / 2$, it holds

$$
\begin{aligned}
\cos ^{2} \theta & =\frac{a-\lambda_{-}}{\lambda_{+}-\lambda_{-}}=\frac{a-c+\sqrt{(a-c)^{2}+4|b|^{2}}}{2 \sqrt{(a-c)^{2}+4|b|^{2}}} \geq \frac{a-c+\sqrt{(a-c)^{2}+4|b|^{2}}}{2 \sqrt{(a+c)^{2}-4\left(a c-|b|^{2}\right)}} \\
& \geq \frac{a-c+\sqrt{(a-c)^{2}}}{2 \sqrt{(a+c)^{2}}}=\frac{a-c}{a+c} \geq \frac{a / 2}{2 a}=\frac{1}{4} .
\end{aligned}
$$

Then we get

$$
\frac{1}{4} a^{2 / p} \leq \lambda_{+}^{2 / p} \cos ^{2} \theta \leq a_{p} \leq \lambda_{+}^{2 / p} \leq 2^{2 / p} a^{2 / p}
$$

and

$$
c_{p} \geq \lambda_{-}^{2 / p} \geq(1-\sqrt{1-\varepsilon})^{2 / p} c^{2 / p} .
$$

Now we are going to estimate an upper bound for $c_{p}$.

By (10) and taking into account that $2 \sqrt{(a-c)^{2}+4|b|^{2}} \geq 2(a-c) \geq a$ (we have used here the hypothesis $c<a / 2$ ), we have

$$
\begin{aligned}
2^{2 / p} \frac{c_{p}}{c^{2 / p}}= & 2^{2 / p} \frac{\lambda_{+}^{2 / p} \sin ^{2} \theta+\lambda_{-}^{2 / p} \cos ^{2} \theta}{c^{2 / p}} \\
= & \left(\frac{a+c+\sqrt{(a-c)^{2}+4|b|^{2}}}{c}\right)^{2 / p} \cdot \frac{-a+c+\sqrt{(a-c)^{2}+4|b|^{2}}}{2 \sqrt{(a-c)^{2}+4|b|^{2}}} \\
& +\left(\frac{a+c-\sqrt{(a-c)^{2}+4|b|^{2}}}{c}\right)^{2 / p} \cdot \frac{a-c+\sqrt{(a-c)^{2}+4|b|^{2}}}{2 \sqrt{(a-c)^{2}+4|b|^{2}}} \\
\leq & \left(\frac{a+c+\sqrt{(a-c)^{2}+4(1-\varepsilon) a c}}{c}\right)^{2 / p} \cdot \frac{-a+c+\sqrt{(a-c)^{2}+4(1-\varepsilon) a c}}{a} \\
& +\left(\frac{\left.a+c-\sqrt{(a-c)^{2}}\right)^{2 / p} \cdot \frac{a-c}{c}+\sqrt{(a-c)^{2}+4(1-\varepsilon) a c}}{a}\right. \\
= & \left(\frac{a+c+\sqrt{(a-c)^{2}+4(1-\varepsilon) a c}}{c}\right)^{2 / p} \cdot \frac{-a+c+\sqrt{(a-c)^{2}+4(1-\varepsilon) a c}}{a} \\
& +2^{2 / p \frac{a-c+\sqrt{(a-c)^{2}+4(1-\varepsilon) a c}}{a}} \cdot
\end{aligned}
$$

Notice that $c_{p} \leq 2^{-2 / p} f_{p}(a / c) c^{2 / p}$, where

$$
\begin{aligned}
f_{p}(s) & :=\left(s+1+\sqrt{(s-1)^{2}+4(1-\varepsilon) s}\right)^{2 / p}\left(-1+\frac{1}{s}+\sqrt{\left(1-\frac{1}{s}\right)^{2}+4(1-\varepsilon) \frac{1}{s}}\right) \\
& +2^{2 / p}\left(1-\frac{1}{s}+\sqrt{\left(1-\frac{1}{s}\right)^{2}+4(1-\varepsilon) \frac{1}{s}}\right)
\end{aligned}
$$

and we just need to prove that $\sup _{s \geq 1} f_{p}(s)<\infty$. This is clear because $f_{p}$ is continuous on $[1, \infty)$ and $\lim _{s \rightarrow \infty} f_{p}(s)<\infty$, since $p \geq 2$ and 
as $s \rightarrow \infty$.

$$
\begin{aligned}
& \left(s+1+\sqrt{(s-1)^{2}+4(1-\varepsilon) s}\right)^{2 / p} \approx(2 s)^{2 / p}, \\
& -1+\frac{1}{s}+\sqrt{\left(1-\frac{1}{s}\right)^{2}+4(1-\varepsilon)} \frac{1}{s} \approx \frac{2(1-\varepsilon)}{s}, \\
& 1-\frac{1}{s}+\sqrt{\left(1-\frac{1}{s}\right)^{2}+4(1-\varepsilon) \frac{1}{s}} \approx 2,
\end{aligned}
$$

Then, we have proved, in both cases, that $a_{p} \asymp a^{2 / p}$ and $c_{p} \asymp c^{2 / p}$.

Finally, we will prove that the norms are equivalent.

Notice that, for every $z, w \in \mathbb{C}$,

$$
(z, w) V^{2 / p}(z, w)^{*}=a_{p}|z|^{2}+2 \Re\left(b_{p} z \bar{w}\right)+c_{p}|w|^{2} .
$$

Hence,

$$
(z, w) V^{2 / p}(z, w)^{*} \leq a_{p}|z|^{2}+2 \sqrt{a_{p} c_{p}}|z||w|+c_{p}|w|^{2} \leq 2\left(a_{p}|z|^{2}+c_{p}|w|^{2}\right) .
$$

Besides, $\left|2 \Re\left(b_{p} z \bar{w}\right)\right| \leq 2 \sqrt{(1-\varepsilon) a_{p} c_{p}}|z||w|$ since $V^{2 / p}$ is elliptic. Then,

$$
(z, w) V^{2 / p}(z, w)^{*} \geq a_{p}|z|^{2}-2 \sqrt{(1-\varepsilon) a_{p} c_{p}}|z||w|+c_{p}|w|^{2} \geq(1-\sqrt{1-\varepsilon})\left(a_{p}|z|^{2}+c_{p}|w|^{2}\right) .
$$

Therefore, by (12) and (13), for every polynomial $q$

$$
(1-\sqrt{1-\varepsilon})^{p / 2}\left(a_{p}^{p / 2}|q|^{p}+c_{p}^{p / 2}\left|q^{\prime}\right|^{p}\right) \leq\left(\left(q, q^{\prime}\right) V^{2 / p}\left(q, q^{\prime}\right)^{*}\right)^{p / 2} \leq 2^{p / 2} 2^{p / 2-1}\left(a_{p}^{p / 2}|q|^{p}+c_{p}^{p / 2}\left|q^{\prime}\right|^{p}\right) .
$$

We finish the proof combining the inequalities above with the fact that $a_{p} \asymp a^{2 / p}$ and $c_{p} \asymp c^{2 / p}$.

The thesis of Theorem 2.3 does not hold when $1 \leq p<2$, but it is possible to obtain a similar result requiring the ellipticity of $V^{2}$ instead of the ellipticity of $V$.

Theorem 2.4. If $V^{2}$ is elliptic, then $V^{2 / p}$ is also elliptic and the norms $W^{1, p}(V \mu)$ and $W^{1, p}((a+|b|) \mu,(c+$ $|b|) \mu$ ) are equivalent on the space of polynomials for every $1 \leq p<2$.

Remark 2.5. In fact, the thesis of Theorem 2.4 holds for every $1 \leq p<\infty$, but the case $2 \leq p<\infty$ is more efficiently covered by Theorem 2.3.

Proof. The same proof of Theorem 2.3 is valid replacing $V$ by

$$
V_{1}:=V^{2}=\left(\begin{array}{ll}
a_{1} & b_{1} \\
\bar{b}_{1} & c_{1}
\end{array}\right)=\left(\begin{array}{cc}
a^{2}+|b|^{2} & (a+c) b \\
(a+c) \bar{b} & c^{2}+|b|^{2}
\end{array}\right),
$$

since $V_{1}=U D U^{*}$, with $D=\Lambda^{2}, V^{2 / p}=U D^{1 / p} U^{*}$ and $1 / p \leq 1$.

With this argument we prove that $a_{p} \asymp a_{1}^{1 / p}$ and $c_{p} \asymp c_{1}^{1 / p}$, and that the norms $W^{1, p}(V \mu)$ and $W^{1, p}\left(a_{1}^{1 / 2} \mu, c_{1}^{1 / 2} \mu\right)$ are equivalent on the space of polynomials for every $1 \leq p<\infty$.

Since $a_{1}=a^{2}+|b|^{2}$ and $c_{1}=c^{2}+|b|^{2}$, the norms $W^{1, p}\left(a_{1}^{1 / 2} \mu, c_{1}^{1 / 2} \mu\right)$ and $W^{1, p}((a+|b|) \mu,(c+|b|) \mu)$ are equivalent on the space of polynomials for every $1 \leq p<\infty$.

Remark 2.6. For the sake of simplicity, every following result will be stated just in the case $2 \leq p<\infty$. However, all of them hold for $1 \leq p<2$, requiring the ellipticity of $V^{2}$ instead of the ellipticity of $V$, and replacing the hypotheses on $a \mu$ and $c \mu$ by the corresponding hypotheses on $(a+|b|) \mu$ and $(c+|b|) \mu($ it suffices to use Theorem 2.4 instead of Theorem 2.3).

The following result is a direct consequence of Theorems 2.2 and 2.3.

Theorem 2.7. Let us assume that $S(\mu)$ is compact, $V$ is elliptic and $p \geq 2$. Then, the multiplication operator is bounded in $W^{1, p}(V \mu)$ if and only if (5) takes place.

The latter theorem and Theorem 2.1 give the following result.

Theorem 2.8. Let us assume that $S(\mu)$ is compact, $V$ is elliptic, $p \geq 2$ and (5) takes place. Let $\left\{q_{n}\right\}_{n \geq 0}$ be a sequence of extremal polynomials with respect to (1). Then the zeros of $\left\{q_{n}\right\}_{n \geq 0}$ lie in the bounded disk $\{z:|z| \leq 2\|M\|\}$. 
In general, it is not difficult to check wether or not (5) holds. It is clear that if there exists a constant $C$ such that $c \leq C a \mu$-almost everywhere, then (5) holds. In [31] and [32] some other very simple conditions implying (5) are shown.

The following direct corollary of Theorem 2.8 is a stronger version of Theorem 1.2.

Corollary 2.9. If $S(\mu)$ is compact, $V$ is elliptic, $p \geq 2$ and $c \leq C a$, $\mu$-almost everywhere for some constant $C$, then the zeros of $\left\{q_{n}\right\}_{n \geq 0}$ lie in the bounded disk $\{z:|z| \leq 2\|M\|\}$.

We finish this section with the following result, which shows that the ellipticity of $V$ (or of $V^{2}$ ) is a weaker condition than (2) in Theorem 1.1 .

Proposition 2.10. If (2) holds, then $V^{2 / p}$ is elliptic for every $1 \leq p<\infty$, with $\varepsilon=2 /\left(1+C^{2 / p}\right)$. Furthermore, the converse is false.

Proof. Assume that $\lambda_{0} \leq C \lambda_{1}$ and $\lambda_{1} \leq C \lambda_{0}$. Notice that

$$
\begin{aligned}
\frac{\sin ^{2} 2 \theta+(1-\varepsilon)\left(1+\cos ^{2} 2 \theta\right)}{\varepsilon \sin ^{2} 2 \theta} & =\frac{2-\varepsilon\left(1+\cos ^{2} 2 \theta\right)}{\varepsilon \sin ^{2} 2 \theta}=\frac{2}{\varepsilon \sin ^{2} 2 \theta}-\frac{1+\cos ^{2} 2 \theta}{\sin ^{2} 2 \theta} \\
& =\left(\frac{1}{\varepsilon}-1\right) \frac{2}{\sin ^{2} 2 \theta}+\frac{2}{\sin ^{2} 2 \theta}-\frac{1+\cos ^{2} 2 \theta}{\sin ^{2} 2 \theta} \\
& =\left(\frac{1}{\varepsilon}-1\right) \frac{2}{\sin ^{2} 2 \theta}+1 \geq \frac{2}{\varepsilon}-1 .
\end{aligned}
$$

Now, taking $\varepsilon=2 /\left(1+C^{2 / p}\right)$, we have

$$
\frac{\sin ^{2} 2 \theta+(1-\varepsilon)\left(1+\cos ^{2} 2 \theta\right)}{\varepsilon \sin ^{2} 2 \theta} \geq \frac{2}{\varepsilon}-1=C^{2 / p} \geq \frac{1}{2}\left(\frac{\lambda_{0}}{\lambda_{1}}\right)^{2 / p}+\frac{1}{2}\left(\frac{\lambda_{1}}{\lambda_{0}}\right)^{2 / p} .
$$

In the proof of Theorem 2.3 we have seen the latter inequality is equivalent to $\left|b_{p}\right|^{2} \leq(1-\varepsilon) a_{p} c_{p}$ (see (11)). Therefore $V^{2 / p}$ is elliptic for every $1 \leq p<\infty$.

Consider now $a$ and $c$ such that either $a / c$ or $c / a$ are not in $L^{\infty}(\mu)$, and $b=0$. It is straightforward that $V^{2 / p}$ is elliptic for every $1 \leq p<\infty$, since it is a diagonal matrix, but that the inequality (2) does not hold, since $\lambda_{0}=a$ and $\lambda_{1}=c$.

The following example shows how Theorem 1.2 improves in a fundamental way the earlier results in the literature (Theorems 1.1 and 2.2).

Example 2.11. Let us consider any finite measure $\mu$ with support $S(\mu) \subseteq[0,1]$, a function $\eta$ with $\|\eta\|_{L^{\infty}(\mu)}<$ 1 , and

$$
V:=\left(\begin{array}{cc}
x^{\alpha} & \overline{\eta(x)} x^{\beta} \\
\eta(x) x^{\beta} & x^{\gamma}
\end{array}\right)
$$

Since $V$ is a positive definite matrix, we have $2 \beta \geq \alpha+\gamma$, and this inequality also guarantees the ellipticity of $V$.

Theorem 1.1 gives that the zeros of extremal polynomials are bounded when $\alpha=\gamma$, but Theorem 1.2 assures that, in order to have the boundedness, it is sufficient to have $\alpha \leq \gamma$.

Although Theorem 2.2 also assures that the zeros of extremal polynomials are bounded when $\alpha \leq \gamma$, it requires the additional hypothesis $\eta=0 \mu$-almost everywhere.

\section{Asymptotic of eXtremal polynomials}

The class Reg of regular measures is defined in [35]. For measures supported on a compact set of the complex plane, the authors prove that (see Theorem 3.1.1) $\mu \in \mathbf{R e g}$ if and only if

$$
\lim _{n \rightarrow \infty}\left\|Q_{n}\right\|_{L^{2}(\mu)}^{1 / n}=\operatorname{cap}(S(\mu)),
$$

where $Q_{n}$ denotes the $n$th monic orthogonal polynomial (in the standard sense) with respect to $\mu,\|\cdot\|_{L^{2}(\mu)}$ is the usual norm in the space $L^{2}(\mu)$ of square integrable functions with respect to $\mu$ and $\operatorname{cap}(S(\mu)) \operatorname{denotes}$ the logarithmic capacity of $S(\mu)$. 
Given a polynomial $q$ whose degree is exactly $n$, we define the normalized zero counting measure of $q$ as

$$
\nu(q):=\frac{1}{n} \sum_{j=1}^{n} \delta_{z_{j}},
$$

where $z_{1}, z_{2}, \cdots, z_{n}$ are the zeros of $q$ repeated according to their multiplicity, and $\delta_{z_{j}}$ is the Dirac measure with mass one at the point $z_{j}$.

By $\omega_{S(\mu)}$ we denote the equilibrium measure of $S(\mu)$.

Theorem 3.1. Let us assume that a $\mu \in \mathbf{R e g}, S(\mu)$ is regular with respect to the Dirichlet problem, $\left\{q_{n}\right\}_{n \geq 0}$ is the sequence of extremal polynomials with respect to $\|\cdot\|_{W^{1, p}(V \mu)}$ (where $V$ is elliptic and $\left.2 \leq p<\infty\right)$ and (5) takes place. Then,

$$
\lim _{n \rightarrow \infty}\left\|q_{n}^{(j)}\right\|_{S(\mu)}^{1 / n}=\operatorname{cap}(S(\mu)), \quad j \geq 0
$$

Furthermore, if $S(\mu)$ has empty interior and its complement is connected, then

$$
\lim _{n \rightarrow \infty} \nu\left(q_{n}^{(j)}\right)=\omega_{S(\mu)}, \quad j \geq 0 .
$$

in the weak star topology of measures.

Proof. It is sufficient to follow the proof offered in [20, Theorem 2], taking into consideration that the hypothesis removed in our theorem are equivalent to the fact that, in our context, the multiplication operator is bounded (see Theorem 2.2) and the norms of $W^{1, p}(V \mu)$ and $W^{1, p}(a \mu, c \mu)$ are equivalent (see Theorem $2.3)$.

Let $\Omega$ be the unbounded component of the complement of $S(\mu)$. We denote by $g_{\Omega}(z ; \infty)$ the Green's function for $\Omega$ with logarithmic singularity at $\infty$. If $S(\mu)$ is regular with respect to the Dirichlet problem, then $g_{\Omega}(z ; \infty)$ is continuous up to the boundary and it can be extended continuously to all $\mathbb{C}$, with value zero on $\mathbb{C} \backslash \Omega$.

Theorem 3.2. Let us assume that a $\mu \in \mathbf{R e g}, S(\mu)$ is compact and regular with respect to the Dirichlet problem, $\left\{q_{n}\right\}_{n \geq 0}$ is the sequence of extremal polynomials with respect to $\|\cdot\|_{W^{1, p}(V \mu)}$ (where $V$ is elliptic and $2 \leq p<\infty)$ and (5) takes place. Then, for each $j \geq 0$,

$$
\limsup _{n \rightarrow \infty}\left|q_{n}^{(j)}(z)\right|^{1 / n} \leq \operatorname{cap}(S(\mu)) e^{g_{\Omega}(z ; \infty)},
$$

uniformly on compact subsets of $\mathbb{C}$. Furthermore,

$$
\lim _{n \rightarrow \infty}\left|q_{n}^{(j)}(z)\right|^{1 / n}=\operatorname{cap}(S(\mu)) e^{g_{\Omega}(z ; \infty)},
$$

uniformly on each compact subset of $\{z:|z|>2\|M\|\} \cap \Omega$. Finally, if the interior of $S(\mu)$ is empty and its complement connected, we have equality in (14) for all $z \in \mathbb{C}$, except on a set of capacity zero, $S\left(\omega_{S(\mu)}\right) \subset\{z:|z| \leq 2\|M\|\}$ and,

$$
\lim _{n \rightarrow \infty} \frac{q_{n}^{(j+1)}(z)}{n q_{n}^{(j)}(z)}=\int \frac{d \omega_{S(\mu)}(x)}{z-x}
$$

uniformly on each compact subset of $\{z:|z|>2\|M\|\}$.

Proof. The proof in [20, Theorem 6] is valid taking into account again that, in our context, the multiplication operator is bounded (see Theorem 2.2) and the norms of $W^{1, p}(V \mu)$ and $W^{1, p}(a \mu, c \mu)$ are equivalent (see Theorem 2.3).

\section{REFERENCES}

[1] V. Alvarez, D. Pestana, J. M. Rodríguez, E. Romera, Weighted Sobolev spaces on curves, J. Approx. Theory 119 (2002) 41-85.

[2] A. Branquinho, A. Foulquié, F. Marcellán, Asymptotic behavior of Sobolev type orthogonal polynomials on a rectifiable Jordan curve or arc, Constr. Approx. 18 (2002), 161-182.

[3] R. C. Brown, B. Opic, Embeddings of weighted Sobolev spaces into spaces of continuous functions, Proc. R. Soc. Lond. A 439 (1992), 279-296.

[4] A. Cachafeiro, F. Marcellán, Orthogonal polynomials of Sobolev type on the unit circle, J. Approx. Theory 78 (1994), $127-146$.

[5] E. W. Cheney, Introduction to approximation theory, third ed., A.M.S. Chelsea, Providence, RI, 2000.

[6] P. J. Davis, Interpolation and approximation, Dover, New York, 1975. 
[7] B. Della Vecchia, G. Mastroianni, J. Szabados, Approximation with exponential weights in [-1,1], J. Math. Anal. Appl. 272 (2002), 1-18.

[8] W. N. Everitt, L. L. Littlejohn, The density of polynomials in a weighted Sobolev space, Rendiconti di Matematica, Serie VII, 10 (1990), 835-852.

[9] W. N. Everitt, L. L. Littlejohn, S. C. Williams, Orthogonal polynomials in weighted Sobolev spaces, Lecture notes in pure and applied mathematics, 117 (1989), Marcel Dekker, 53-72.

[10] W. N. Everitt, L. L. Littlejohn, S. C. Williams, Orthogonal polynomials and approximation in Sobolev spaces, J. Comput. Appl. Math. 48 (1993), 69-90.

[11] A. Foulquié, F. Marcellán, K. Pan, Asymptotic behavior of Sobolev-type orthogonal polynomials on the unit circle, J. Approx. Theory 100 (1999), 345-363.

[12] J. Heinonen, T. Kilpeläinen, O. Martio, Nonlinear potential theory of degenerate elliptic equations, Oxford Science Publ., Clarendon Press, 1993.

[13] A. Iserles, P. E. Koch, S. P. Norsett, J. M. Sanz-Serna, Orthogonality and approximation in a Sobolev space, in Algorithms for Approximation. J. C. Mason and M. G. Cox, Chapman \& Hall, London, 1990.

[14] A. Iserles, P. E. Koch, S. P. Norsett, J. M. Sanz-Serna, On polynomials orthogonal with respect to certain Sobolev inner products, J. Approx. Theory 65 (1991), 151-175.

[15] T. Kilpeläinen, Weighted Sobolev spaces and capacity, Ann. Acad. Sci. Fennicae, Series A. I. Math. 19 (1994), 95-113.

[16] A. Kufner, Weighted Sobolev spaces, Teubner Verlagsgesellschaft, Teubner-Texte zur Mathematik (Band 31), Leipzig, 1980. Also published by John Wiley \& Sons, New York, 1985.

[17] A. Kufner, B. Opic, How to define reasonably weighted Sobolev Spaces, Commentationes Mathematicae Universitatis Caroline 25(3) (1984), 537-554.

[18] A. Kufner, A. M. Sändig, Some applications of weighted Sobolev spaces, Teubner Verlagsgesellschaft, Teubner-Texte zur Mathematik (Band 100), Leipzig, 1987.

[19] D. S. Lubinsky, Weierstrass' theorem in the twentieth century: a selection, Quaestiones Mathematicae 18 (1995), 91-130.

[20] G. López Lagomasino, I. Pérez Izquierdo, H. Pijeira, Asymptotic of extremal polynomials in the complex plane, J. Approx. Theory 137 (2005), 226-237.

[21] G. López Lagomasino, H. Pijeira, Zero location and $n$-th root asymptotics of Sobolev orthogonal polynomials, J. Approx. Theory 99 (1999), 30-43.

[22] G. López Lagomasino, H. Pijeira, I. Pérez, Sobolev orthogonal polynomials in the complex plane, J. Comp. Appl. Math. 127 (2001), 219-230.

[23] Martínez-Finkelshtein, A., Bernstein-Szegö's theorem for Sobolev orthogonal polynomials. Constr. Approx. 16, 73-84 (2000)

[24] V. G. Maz'ja, Sobolev spaces, Springer-Verlag, New York, 1985.

[25] B. Muckenhoupt, Hardy's inequality with weights, Studia Math. 44 (1972), 31-38.

[26] A. Pinkus, Weierstrass and approximation theory, J. Approx. Theory 107 (2000), 1-66.

[27] J. M. Rodríguez, V. Alvarez, E. Romera, D. Pestana, Generalized weighted Sobolev spaces and applications to Sobolev orthogonal polynomials I, Acta Appl. Math. 80 (2004), 273-308.

[28] J. M. Rodríguez, V. Alvarez, E. Romera, D. Pestana, Generalized weighted Sobolev spaces and applications to Sobolev orthogonal polynomials II, Approx. Theory and its Appl. 18:2 (2002), 1-32.

[29] J. M. Rodríguez, V. Alvarez, E. Romera, D. Pestana, Generalized weighted Sobolev spaces and applications to Sobolev orthogonal polynomials: a survey, Electr. Trans. Numer. Anal. 24 (2006), 88-93.

[30] J. M. Rodríguez, The multiplication operator in Sobolev spaces with respect to measures, J. Approx. Theory 109 (2001), 157-197.

[31] J. M. Rodríguez, A simple characterization of weighted Sobolev spaces with bounded multiplication operator, J. Approx. Theory 153 (2008) 53-72.

[32] J. M. Rodríguez, J. M. Sigarreta, Sobolev spaces with respect to measures in curves and zeros of Sobolev orthogonal polynomials, Acta Appl. Math. 104 (2008), 325-353.

[33] W. Rudin, Real and complex analysis (Second Edition), McGraw-Hill, 1974.

[34] E. B. Saff, V. Totik, Logarithmic potentials with external fields, Grundlehren der mathematischen Wissenschaften 316, Springer-Verlag, 1998.

[35] H. Stahl, V. Totik, General orthogonal polynomials, Cambridge University Press, Cambridge, 1992.

St. Louis University (Madrid Campus), Avenida del Valle 34, 28003 Madrid, Spain

E-mail address: aportil2@slu.edu

Departamento de Matemáticas, Universidad Carlos IiI de Madrid, Avenida de la Universidad 30, 28911 Leganés, MADRID, SPAIN

E-mail address: jomaro@math.uc3m.es

Departamento de Matemáticas, Universidad Carlos III de Madrid, Avenida de la Universidad 30, 28911 Leganés, MADRID, SPAIN

E-mail address: etouris@math.uc3m.es 